\title{
Multi-physics and Multi-scale Modelling in Cardiovascular Physiology: Advanced User Methods for Simulation of Biological Systems with ANSYS/CFX
}

\author{
V. Díaz-Zuccarini ${ }^{1}$, D. Rafirou ${ }^{2}$, D.R. Hose ${ }^{1}$, P.V. Lawford ${ }^{1}$, and A.J. Narracott ${ }^{1}$ \\ ${ }^{1}$ University of Sheffield, Academic Unit of Medical Physics, Royal Hallamshire Hospital, \\ Glossop Road, S10 2JF, Sheffield, UK \\ ${ }^{2}$ Electrical Engineering Department / Biomedical Engineering Center, \\ Technical University of Cluj-Napoca, 15, C. Daicoviciu Street, 400020 Cluj-Napoca, Romania \\ v.diaz@sheffield.ac.uk
}

\begin{abstract}
This work encompasses together a number of diverse disciplines (physiology, biomechanics, fluid mechanics and simulation) in order to develop a predictive model of the behaviour of a prosthetic heart valve in vivo. The application of simulation, for the study of other cardiovascular problems, such as blood clotting is also discussed. A commercial, finite volume, computational fluid dynamics (CFD) code (ANSYS/CFX) is used for the 3D component of the model. This software provides technical options for advanced users which allow userspecific variables to be defined that will interact with the flow solver. Userdefined functions and junction boxes offer appropriate options to facilitate multi-physics and multi-scale complex applications. Our main goal is to present a 3D model using the internal features available in ANSYS/CFX coupled to a multiscale model of the left ventricle to address complex cardiovascular problems.
\end{abstract}

Keywords: Multi-scale models, cardiovascular modelling \& simulation, mechanical heart valves, coupled 3D-lumped parameter models, simulation software.

\section{Introduction}

Modelling and simulation can be used to explore complex interactions which occur in the human body. In order to model these biological processes, a multi-scale approach is needed. One of the "success stories" in bioengineering is the study of the fluid dynamics of blood (haemodynamics) within the cardiovascular system and the relationship between haemodynamics and the development of cardiovascular disease [1].

Cardiovascular models present a particular challenge in that they require both a multi-scale and a multi-physics approach. Using finite elements for the whole system is computationally prohibitive thus, a compromise is needed. The most sophisticated fluid-solid interaction structures [2] provide exquisite detail in the fluid domain, but are limited in that the boundary conditions are prescribed. An alternative multiscale solution is to couple lumped parameter models of the boundary conditions with a finite element model of the part where detail and accuracy are needed [3]. Significant improvement can be made in terms of the understanding of the underlying physics if the lumped parameter approach includes more physiologically representative 
mechanisms rather than traditional "black-box" models. Usually, boundary conditions are expressed in terms of pressure and flow. These are the macroscopic expression of physiological or pathological conditions. These macroscopic variables can be related to the microscopic level of the physiology/pathology through molecular/cellular aspects of the process with the definition of a new range of variables. These new variables will not be available to the user as normal boundary conditions, as they are totally dependant on the level of modelling chosen for the problem under study.

\section{ANSYS/CFX Special Features: Crossing the Flow Interface Towards Biological Modelling}

ANSYS/CFX is highly specialized software, built in such a way that interaction with specific features of the solver is kept to a minimum. Whilst this is appropriate for many applications, in order to run multi-scale applications, the user must be able to interact with the solver at a lower level. The user needs to be able to define where, when and how the equations and variables imposed as boundaries (i.e. mathematical models) will participate in the solution. In this paper we describe two options which ANSYS/CFX provides for advanced users: Command Expression Language (CEL) functions/subroutines and Junction Boxes. We will use CEL functions/subroutines at the flow interface to solve the equations of the boundary conditions at different scales and Junction Boxes to provide a structure to update the variables.

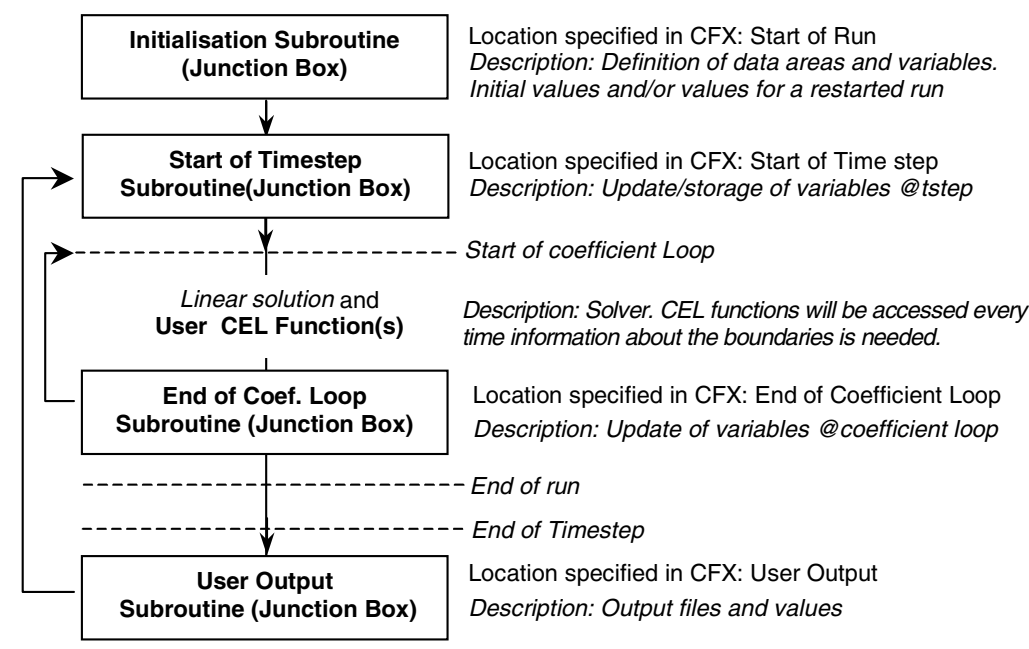

Fig. 1. Structure of an ANSYS/CFX model using Junction Boxes and CEL Subroutines

Definitions [4]:

- CEL Subroutines: These are used in conjunction with User CEL Functions to define quantities in ANSYS CFX-Pre based on a FORTRAN subroutine. The User CEL Routine is used to set the calling name, the location of the Subroutine and the location of the Shared Libraries associated with the Subroutine. 
- CEL Functions: User CEL Functions are used in conjunction with User CEL Routines. User Functions set the name of the User CEL Routine associated with the function, and the input and return arguments.

- Junction Box Routines: Are used to call user-defined FORTRAN subroutines during execution of the ANSYS CFX-Solver. A Junction Box Routine object must be created so that the ANSYS CFX-Solver knows the location of the Subroutine, the location of its Shared Libraries and when to call the Subroutine.

A general form of a transient and structured model in ANSYS/CFX is shown in Fig. 1. When solving differential or partial differential equation models as boundary conditions for an ANSYS/CFX model the equations describing the boundary condition must be discretized in such a way that variables are solved and passed to ANSYS/CFX at each time-step and updated within coefficient loops. Using ANSYS/CFX functions, the value of physical quantities at specific locations of the 3D modelare passed as arguments to the boundary condition model and a specific value is returned to ANSYS/CFX to update the boundary condition for that region. Using this approach, the boundary conditions of the 3D model are updated at each coefficient loop of the flow solver, providing close coupling of the 3D ANSYS/CFX model and the boundary condition model.

\section{Case Study: Coupling a Model of the Left Ventricle to an Idealized Heart Valve}

This section describes the development of more complex boundary conditions to represent the behaviour of the left ventricle (LV). We also describe the coupling of the LV model to a fluid-structure interaction (FSI) model of an idealized heart valve.

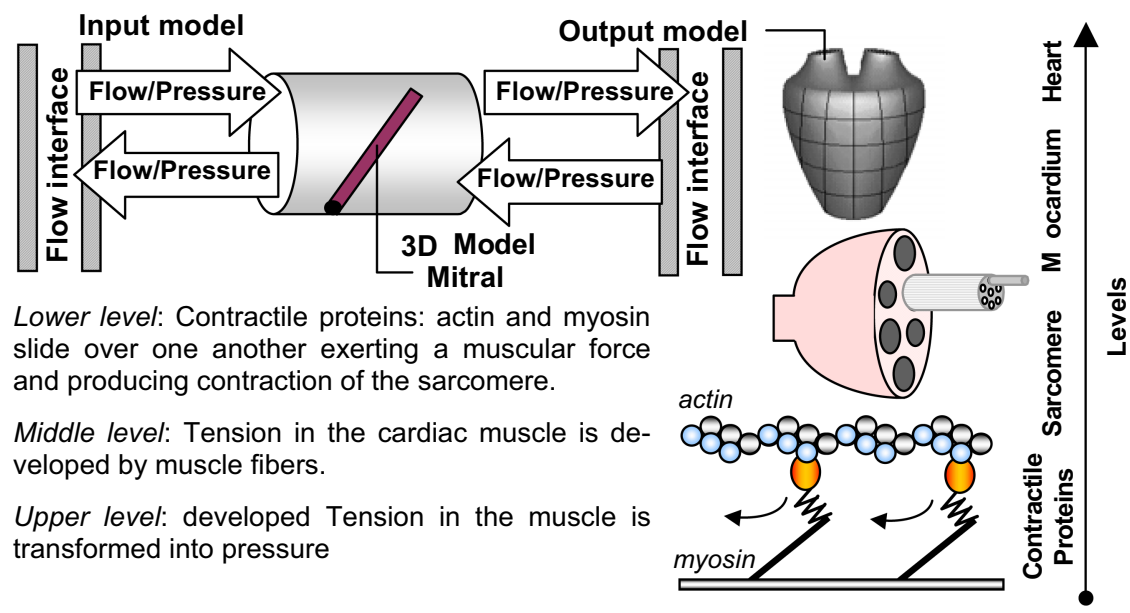

Fig. 2. Representation of the LV model and its different physical scales. Cardiac contraction starts at protein level in the sarcomere. Protein contraction produces tension in the cardiac wall that then is translated into pressure. 
Fig. 2 shows the sub-levels of organisation of the ventricle that are taken into account to give a "more physiologically realistic" model of the LV. Following the procedure described in ex vivo isolated LV preparations, a constant pressure source is connected to the ventricle via the mitral valve (input model). The blood fills the LV (output model) and ejects a volume of blood into the arterial network via the aortic valve. Contraction in the cardiac muscle is described starting from the microscopic level of contractile proteins (actin and myosin), up to the tissue (muscle level) and to the LV level to finally reach the haemodynamic part of the LV and its arterial load.

\subsection{A Multi-scale Model of the Left Ventricle}

The contractile proteins (actin and myosin) acting at the level of the sarcomere produce cardiac contraction. These slide over each other [5], attaching and detaching by means of cross-bridges, to produce shortening of the sarcomere and hence contraction of the ventricular wall (Fig. 2). To represent the chemical reaction from the detached state $\left(X_{D}\right)$ to the attached one $\left(X_{A}\right)$ and vice-versa we use the simplest model available:

$$
X \underset{D \stackrel{k_{d}}{\rightarrow}}{\stackrel{k_{a}}{\rightarrow}} X_{A}
$$

The periodically varying kinetic constants of attachment and detachment are $k_{a}$ and $k_{d}$. The chemical potential of a thermodynamic system is the amount by which the energy of the system would change if an additional particle were introduced, with the entropy and volume held fixed. The modified Nernst formula provides a first approximation:

$$
\begin{gathered}
\mu_{a}=\left(A_{a}+B_{a}(T) \ln \left(X_{A}\right)\right) \beta\left(l_{m}\right) \\
\mu_{d}=A_{d}+B_{d}(T) \ln \left(X_{D}\right)
\end{gathered}
$$

The only non-classical feature is the $\beta$ factor of equation (2). For a more detailed explanation, the reader is referred to [6],[7]. Using the usual expression of the reaction rate in chemical thermodynamics, the reaction flow may be expressed as:

$$
\frac{d X_{A}}{d t}=k_{A}(t) \cdot e^{\left(\mu_{d}-A_{d}\right) / B_{d}}-k_{D}(t) \cdot e^{\left(\mu_{a}-\beta A_{a}\right) / B a}
$$

This can be reduced to the following expression:

$$
\frac{d X_{A}}{d t}=k_{A}(t)\left(1-X_{A}(t)\right)-k_{D}(t) X_{D}(t)^{\beta}
$$

We can now relate the chemical and mechanical aspects of the model, to describe the chemo-mechanic transduction in the cardiac muscle. A single muscle equivalent to all the muscles of the heart is considered. Its length is $l_{m}$ and $X_{A}$ is its number of attached cross bridges. Neglecting the energy in free cross bridges, the energy of the muscle is given by:

$$
E_{m}=E_{m}\left(l_{m}, X_{A}\right)
$$


The mechanical power is obtained by derivation:

$$
P_{m}=\frac{\partial E_{m}}{\partial l_{m}} \frac{d l_{m}}{d t}+\frac{\partial E_{m}}{\partial X_{a}} \frac{d X_{A}}{d t}=f_{m} v_{m}+\mu_{a} \dot{X}_{A}
$$

As shown in equation (7), the mechanical force is obtained by derivation of the energy equation of the muscle with respect to the attached cross-bridges $\left(E_{X}\right)$ and the length of muscle fibers $\left(l_{m}\right)$.

$$
f_{m}=E_{X}\left(X_{A}\right) E_{a}^{\prime}\left(l_{m}\right)+\left(1-E_{X}\left(X_{A}\right)\right) E_{r}^{\prime}\left(l_{m}\right)
$$

$E^{\prime}{ }_{a}$ and $E_{r}{ }_{r}$ are respectively, the derivative of the energy of the muscle in active $(a)$ and resting $(r)$ state respect to $l_{m}$ and they are expressed as functions of the muscular force $f_{m}$. Full details of the formulation and assumptions may be found in [6],[7]. In our model, the cardiac chamber transforms muscular force into pressure and volumetric flow into velocity of the muscle. We assume an empiric relationship $\varphi_{\mathrm{G}}$ between volume $Q_{L V}$ and equivalent fibre length $l_{m}$

$$
Q_{L V}(t)=\varphi_{G}\left(l_{m}(t)\right) \leftrightarrow l_{m}(t)=\varphi_{G}^{-1}\left(Q_{L V}(t)\right)
$$

Assuming $\varphi_{G}$ is known and by differentiation of (9), a relationship between the velocity of the muscle $d l_{m} / d t$ and the ventricular volumetric flow $d Q_{L V} / d t$ is obtained:

$$
\frac{d Q_{l v}(t)}{d t}=\psi_{G} \frac{d l_{m}}{d t}
$$

Where $\psi_{\mathrm{G}}=\psi_{\mathrm{G}}\left(Q_{L V}\right)$ is the derivative of $\varphi_{\mathrm{G}}$ with respect to $l_{m}$. If the transformation is power-conservative, mechanical power (depending on muscular force $f_{m}$ ) =hydraulic power (depending on pressure $P$ ).

$$
P \cdot \frac{d Q_{l v}}{d t}=N \cdot f_{m} \cdot \frac{d l_{m}}{d t}
$$

Where we have supposed that pressure $P$ is created from the forces of $N$ identical fibres. Equation (12) is then obtained:

$$
P=\frac{1}{\psi_{G}\left(l_{m}\right)} N \cdot f_{m}
$$

A simple multi-scale model of the left ventricle has been presented, starting from the mechanisms of cardiac contractions at the protein scale. In our case, the ventricular pressure $P$ defined (12) will be the input to the 3D model shown in the next section.

\subsection{Computational Model of the Mitral Valve and Coupling with the LV Model}

The computational approach presented here is based on the use of a fluid-structure interaction model, built within ANSYS/CFX v10. It describes the interaction of a blood-like fluid flowing under a pressure gradient imposed by ventricular contraction, and the structure of a single leaflet valve. A generic CAD model, consisting of a flat leaflet (the occluder) which moves inside a mounting ring (the housing), was built to represent the geometry of the valve (Fig. 3). The thickness of the disc-shaped 
occluder is $\mathrm{h}=2 \mathrm{~mm}$ and its diameter is $\mathrm{d}=21 \mathrm{~mm}$. In the fully closed position, the gap between the mounting ring and the occluder is $\mathrm{g}=0.5 \mathrm{~mm}$. The movement of the occluder is considered to be purely rotational, acting around an eccentric axis situated at distance from its centroid (OX axis). The occluder travels 80 degrees from the fully open to the fully closed position,. To complete the CAD model, two cylindrical chambers were added to represent the atrium and ventricle. The atrial chamber, positioned in the positive OZ direction, is $66 \mathrm{~mm}$ in length whilst the ventricular chamber, positioned in the negative $\mathrm{OZ}$ direction, is $22 \mathrm{~mm}$ in length. Only half of the valve was considered in the 3D model. Blood is considered an incompressible Newtonian fluid with density $\rho=1100 \mathrm{~kg} / \mathrm{m} 3$ and dynamic viscosity $\mu=0.004 \mathrm{~kg} / \mathrm{ms}$.

The unsteady flow field inside the valve is described by the $3 \mathrm{D}$ equations of continuity and momentum (13) with the boundary conditions suggested by Fig. 3 (left).

$$
\rho \frac{\partial \vec{u}}{\partial t}+(\vec{u} \cdot \nabla) \vec{u} \rho=-\nabla p+\mu \Delta \vec{u} ; \nabla \cdot \vec{u}=0
$$

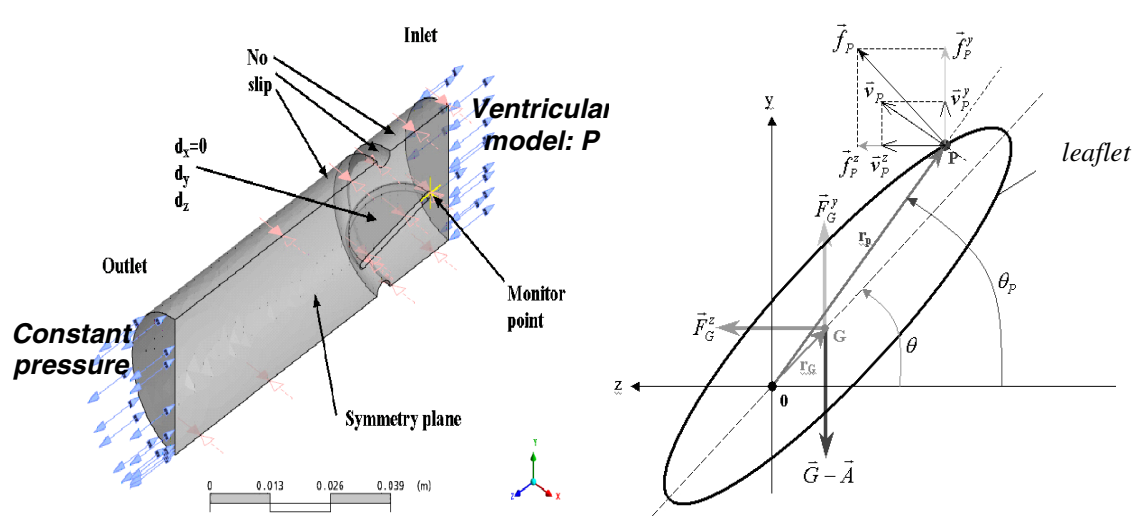

Fig. 3. Left: CAD model and boundary conditions: Snapshot of CFXPre implementation. At the inlet the ventricular pressure $(\mathrm{P})$ is coming from the ventricular model. A constant pressure is applied at the outlet. Right: General representation of a rigid body demonstrating the principles of fluid-structure interaction and rotation of a leaflet. The variables are defined in the text.

The principle used to model the fluid-structure interaction is outlined in Fig 3 (right). The occluder rotates under the combined effects of hydrodynamic, buoyancy and gravitational forces acting on it. At every time step, the drag, $\vec{f}_{P}^{z}$, and lift, $\vec{f}_{P}^{y}$, forces exerted by the flowing fluid on an arbitrary point $\boldsymbol{P}$ of the leaflet's surface, are reduced to the centroid, $\boldsymbol{G}$. The total contribution of drag, $\vec{F}_{G}^{z}$, and lift $\vec{F}_{G}^{y}$ are added to the difference between the gravitational and buoyancy forces. Conservation of the kinetic moment is imposed, resulting in the dynamic equation that describes the motion.

$$
d \theta=\varpi_{\text {old }} d t+\frac{M}{J} d t^{2}
$$


In equation $14, d \theta$ represents the leaflet's angular step, $d t$ is the time step, $\varpi_{\text {old }}$ is the angular velocity at the beginning of the current time step and $M$ is the total momentum acted on the leaflet from the external forces:

$$
M=r_{G}\left[F_{G}^{z} \sin \theta+\left(F_{G}^{y}-G+A\right) \cos \theta\right]
$$

The total moment of inertia is $\mathrm{J}=6 \cdot 10^{-6} \mathrm{~kg} \cdot \mathrm{m}^{2}$.

Some simulation results of the model are shown in Figure 4:
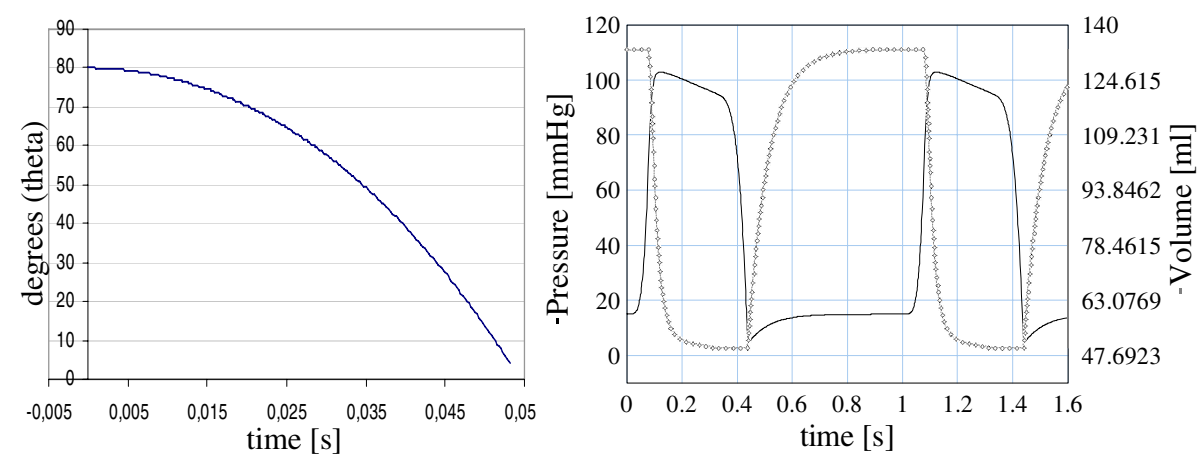

Fig. 4. Left: Closure degree vs. time. Right: Ventricular pressureand Volume vs. time.

It is interesting to notice that from the implementation point of view, closure is continuous and smooth, although when compared to experimental data, closure time is too long [8] and closure is reported too early during systole. This could be due to several reasons, including an insufficient pressure rise and/or an overestimation of the mass of the occluder. This issue is being addressed and will be the object of a future publication. As automatic remeshing tools are not available in the software, remeshing was carried out by hand and the simulation was stopped several times before full closure. Re-start and interpolation tools are available within the software and are appropriate to tackle this particular problem. A restriction in the use of the commercial software chain is that efficient implementation of an automatic remeshing capability is very difficult without close collaboration with the software developers.

\section{Towards Other Biological Applications}

Another feature of the ANSYS/CFX solver enables the user to define additional variables which can be used to represent other properties of the flow field. Previous work described the use of such additional variables to model the change in viscosity of blood during the formation of blood clots [9]. This is based on the work of Friedrich and Reininger [10] who proposed a model of the clotting process based on a variable blood viscosity determined by the residence time of the blood $t$, the viscosity at time $0, \mu_{0}$, and the rate constants $\mathrm{k}_{1}$ and $\mathrm{k}_{2}$, which are dependent on thrombin concentration. Fluid residence time can be modelled within ANSYS/CFX by the introduction of an additional variable which convects throughout the fluid domain. A source term of 1 is defined for the additional variable, resulting in the value of the additional 
variable for each element of the fluid increasing by a unit for every unit of time that it remains within the domain. A model of blood clotting was implemented within CFX 5.5.1 using an additional variable, labelled AGE, to represent the residence time. Due to the method of implementation in CFX, the residence time is expressed as a density and has units of $\mathrm{kgm}^{-3}$. Additional user functions allow the viscosity of the fluid to be defined as a function of the additional variable. Whilst this is a very simple model, it demonstrates the power of custom routines that can be utilized in such modeling. This technique has possible applications for other convective-diffusive processes.

\section{Conclusions and Perspectives}

In this paper, we presented a multi-scale and fully coupled model of the LV with a 3D model of the mitral valve in ANSYS/CFX. The preliminary results are encouraging. Use of advanced features of the software allows describing biological applications. A second application demonstrates the versatility of the software.

\section{Acknowledgement}

The authors acknowledge financial support under the Marie Curie project C-CAReS and thank Dr I. Jones and Dr J. Penrose, ANSYS-CFX, for constructive advice and training.

\section{References}

1. Taylor, C.A., Hughes, T.J., Zarins, C.K. Finite element modeling of three-dimensional pulsatile flow in the abdominal aorta: relevance to atherosclerosis. Annals of Biomedical Engineering (1998) 975-987.

2. De Hart J., Peters G.., Schreurs P., Baaijens F. A three-dimensional computational analysis of fluid-structure interaction in the aortic valve. J. of Biomech, (2003)103-112.

3. Laganà K, Balossino R, Migliavacca F, Pennati G, Bove E.L, de Leval M, Dubini G. Multiscale modeling of the cardiovascular system: application to the study of pulmonary and coronary perfusions in the univentricular circulation. J. of Biomec.(2005)1129-1141.

4. ANSYS/CFX 10.0 manual, ( 2005 ANSYS Europe Ltd (http://www.ansys.com).

5. Huxley AF. Muscle structures and theories of contraction. Prog Biophys. Chem, (1957) 279-305.

6. Díaz-Zuccarini V, ,LeFèvre J. An energetically coherent lumped parameter model of the left ventricle specially developed for educational purposes. Comp in Biol and Med. (In press).

7. Díaz-Zuccarini, V. Etude des Conditions d'efficacite du ventricule gauche par optimisation teleonomique d'un modele de son fonctionnement. PhD Thesis, EC-Lille, (2003)

8. Chandran K. B, Dexter, E. U, Aluri S, Richenbacher W· Negative Pressure Transients with Mechanical Heart-Valve Closure: Correlation between In Vitro and In Vivo Results. Annals of Biomedical Engineering. Volume 26, Number 4 / July, (1998)

9. Narracott A, Smith S, Lawford P, Liu H, Himeno R, Wilkinson I, Griffiths P, Hose R. Development and validation of models for the investigation of blood clotting in idealized stenoses and cerebral aneurysms. J Artif Organs. (2005) 56-62.

10. Friedrich P, Reininger AJ. Occlusive thrombus formation on indwelling catheters: In vitro investigation and computational analysis. Thrombosis and Haemostasis (1995) 66-72. 